\title{
Maturation- and Aging-related Changes in Heat Loss Effector Function
}

\author{
Yoshimitsu Inoue ${ }^{1)}$, Tomoko Kuwahara ${ }^{2)}$ and Tsutomu Araki ${ }^{3)}$ \\ 1) Laboratory for Human Performance Research, Osaka International University \\ 2) Laboratory for Applied Human Physiology, Faculty of Human Development, Kobe University \\ 3) Department of Practical Life Studies, Hyogo University of Teacher Education
}

\begin{abstract}
This paper addresses the ways in which heat loss effector functions change with maturation and aging, using data obtained in our laboratory. Prepubertal children have an underdeveloped sweat function compared with young adults; this is compensated by a greater surface area-to-mass ratio and relatively greater heat loss from cutaneous vasodilation on the head and trunk when the air temperature is lower than the skin temperature. As the heat dissipation depends greatly on the evaporation of sweat, the core temperature of prepubertal children is greater than that of young adults owing to the underdevelopment of sweating. In the elderly the heat loss effector function decreases with aging. The decrease may first involve cutaneous vasodilation, then sweat output per gland, and finally active sweat gland density; and it may proceed from the lower limbs to the back of the upper body, the front of the upper body, then the upper limbs and finally to the head. $J$ Physiol Anthropol Appl Human Sci 23(6): 289-294, 2004 http://www.jstage.jst.go.jp/browse/jpa
\end{abstract}

Keywords: sweating, cutaneous vasodilation, thermoregulation, children, the elderly

\section{Introduction}

Morphological and physiological changes occur with the growth and maturation; the marked changes that occur after middle age are defined as aging. Cutaneous vasodilation and sweating are the two main heat loss responses to the internal and external heat stress in humans. The increased blood flow to the skin (with cutaneous vasodilation) transfers heat from the body core to the periphery; this heat is then dissipated from the skin by conduction, convection, and radiation, as well as by the evaporation of sweat. This review is based on our crosssectional and longitudinal studies of the heat loss in humans and will focus on (1) the development of the heat loss effector function with maturation, (2) the decline of the heat loss effector function with aging, (3) the primary mechanisms underlying the maturation- and aging-related alterations in the heat loss effector function, and (4) the potential to modify these alterations by exercise training.

\section{The development of the heat loss effector function with maturation}

Before considering sweating and blood flow to the skin in children in response to heat, it is important to consider the physical characteristics that affect the heat dissipation. Children have a much greater surface area-to-mass ratio than adults, which allows the children to rely more on dry heat loss than on evaporative heat loss in thermoneutral or warm climates. However, the high surface area-to-mass ratio of children also leads to a higher rate of heat absorption in extremely hot climates. In addition, children have a smaller blood volume than adults do (even when calculated per unit of surface area), which suggests that the heat transferred from the core to the skin is limited in children.

In Japan, the common maxim "a child is a great sweater" is in fact untrue: prepubertal children have a lower total body sweat rate $\left(\mathrm{M}_{\mathrm{sw}}\right)$ and local sweat rate $\left(\mathrm{m}_{\mathrm{sw}}\right)$ than young men do during rest or exercise, regardless of environmental heat stress (Araki et al., 1979; Shibasaki et al., 1997ab, 1999; Inoue et al., 2002). When does the underdeveloped sweat function of children change? Araki et al. (1979) examined $\mathrm{M}_{\mathrm{sw}}$ and chest $\mathrm{m}_{\mathrm{sw}}$ of 40 children at different ages (7-16 years) during light, moderate, and heavy exercise in warm conditions $\left(29^{\circ} \mathrm{C}, 60 \%\right.$ relative humidity $(\mathrm{RH})$ ) and found that both parameters increased significantly between subjects who are less or more than 12-13 years old during exercise at moderate and heavy workloads, but not during light exercise. In the aforementioned study, the subjects who are less than 12 years old did not have pubic hair or a deepening of the voice, whereas those over 13 years old had both features. The aforementioned discrepancy in sweating was greatest under conditions in which much sweating was required to thermoregulate. These results suggest that the sweating is underdeveloped in prepubertal children, possibly owing to the absence of the maturation-associated production of male hormones. Therefore, it is likely that the underdeveloped sweat function in children changes with the onset of puberty.

The magnitude of sweating differs from one region of the 
body to another, and there are regional differences in the degree of the increase in the sweat rate with the increasing of the exercise intensity (Kondo et al., 1998). We compared $\mathrm{m}_{\mathrm{sw}}$ on the forehead, chest, back, forearm, and thigh between prepubertal boys and young men during $30 \mathrm{~min}$ of exercise at 35,50 , and $65 \%$ of maximal oxygen consumption $\left(\mathrm{VO}_{2} \mathrm{max}\right)$ at the air temperature of $28^{\circ} \mathrm{C}$ (Inoue et al., 2002). The magnitude of the increase in rectal temperature $\left(\Delta \mathrm{T}_{\mathrm{re}}\right)$ was similar for the boys and men irrespective of the intensity of the exercise, although the $\Delta \mathrm{T}_{\mathrm{re}}$ in the boys and men increased with the increasing of the exercise intensity. The $\mathrm{m}_{\mathrm{sw}}$ on each of the five sites increased significantly with the increasing of the exercise intensity (except on the thigh of boys), but there was a maturation-related regional difference in the degree of the increase in $\mathrm{m}_{\mathrm{sw}}$. Specifically, boys had a significantly lower $\mathrm{m}_{\mathrm{sw}}$ on the chest and thigh at $50 \% \mathrm{VO}_{2} \max$ and on the forehead, chest, and thigh at $65 \% \mathrm{VO}_{2} \max$, but the $\mathrm{m}_{\mathrm{sw}}$ was not significantly lower on any site at $35 \% \mathrm{VO}_{2}$ max. These findings suggest that the sweat gland ability may not develop at a uniform rate over the whole body during the growth and maturation.

Changes in the $\mathrm{m}_{\mathrm{sw}}$ can result from the changes in the density of heat-activated sweat glands (HASG) and/or the sweat output per gland (SGO). The total number of the sweat glands is fixed by the age of 2-3 years and does not change subsequently. Therefore, prepubertal children, who have relatively smaller total body surface areas, have greater HASG densities. As a consequence, the lower $\mathrm{m}_{\mathrm{sw}}$ observed in the children in the aforementioned study was the result of a lower SGO (Shibasaki et al., 1997ab; Inoue et al., 2002). Sato and Sato (1983) reported that people with lower SGO and functionally inactive sweat glands possess smaller sweat glands and a lower cholinergic sensitivity. These findings raise the possibility that the low $\mathrm{m}_{\mathrm{sw}}$ of children is attributable to the small size of the sweat glands and the reduced cholinergic sensitivity of these glands. Furthermore, regional differences in SGO are affected by maturation (Shibasaki et al., 1997ab; Inoue et al., 2002); that is, men have a greater SGO on the chest compared with the other body sites (e.g., forehead, back, forearm, and thigh), whereas no regional differences are observed in boys. Therefore, it would appear that the increase in SGO that occurs with maturation may not occur at a uniform rate over the entire surface of the body.

During exercise in thermoneutral or warm conditions (air temperature lower than skin temperature), children manifest thermoregulatory responses that depend more on dry heat loss (elevation of skin temperature) than on wet heat loss (sweating) relative to young adults (Araki et al., 1979). The greater elevation of skin temperature observed in children is probably the result of a greater increase in blood flow to the skin than that in young adults.

We used laser Doppler flowmetry to examine blood flow to the skin (SkBF) on the chest, back, and forearm in prepubertal boys and young men who were matched for skinfold thickness and $\mathrm{VO}_{2}$ max per unit mass (Shibasaki et al., 1997b). During moderate exercise $\left(40 \% \mathrm{VO}_{2} \max \right)$ for $45 \mathrm{~min}$ in warm conditions $\left(30^{\circ} \mathrm{C}, 45 \% \mathrm{RH}\right)$, the SkBF increased on the chest and back of the boys more than of the men, but not on the forearm, despite the fact that $\Delta \mathrm{T}_{\mathrm{re}}$ was similar in the boys and men. The age-related differences observed on the chest and back were more marked for cutaneous vascular conductance (CVC; obtained by dividing SkBF by mean arterial pressure (MAP)), because the boys had a lower MAP during exercise. Furthermore, there were no significant differences in SkBF among sites for the boys, whereas, for the men, SkBF was significantly greater on the forearm than on the chest and back. This suggested that there are regional differences in the maturation-related changes in SkBF during exercise. Similar results were reported in our subsequent study in which SkBF was measured on the forehead, chest, and forearm in prepubertal boys and young men during exercise at 35,50 and $65 \% \mathrm{VO}_{2}$ max for $30 \mathrm{~min}$ at an air temperature of $28^{\circ} \mathrm{C}$ (Inoue et al., 2002). We found that SkBF was greater in the boys, not only on the chest, but also on the forehead, even though $\Delta \mathrm{T}_{\mathrm{re}}$ was similar in the prepubertal boys and young men. These results also suggest that during exercise under the aforementioned conditions, the prepubertal boys thermoregulate as efficiently as the young men as a consequence of greater cutaneous vasodilation on the head and trunk, together with a greater surface area-to-mass ratio, despite the lower value of $\mathrm{m}_{\mathrm{sw}}$ in the prepubertal boys. Nevertheless, there are conflicting reports that children exhibit a greater increase in core temperature or heat storage when the ambient temperature is higher than the skin temperature or when the heat radiation is enhanced (Inoue, 2003). That is, the greater cutaneous vasodilation that occurs in prepubertal children cannot offset the lower sweating capacity when the heat dissipation depends greatly on the evaporation of sweat.

Figure 1 shows the characteristics of heat loss responses in prepubertal children. Despite their underdeveloped sweat function, prepubertal children can thermoregulate as efficiently as young adults when the air temperature is lower than the skin temperature; this is a consequence of a greater cutaneous vasodilation on the head and trunk, and a greater surface areato-mass ratio. The greater surface area-to-mass ratio in children leads to a relatively higher rate of heat absorption when the air temperature is higher than the skin temperature. Under such conditions, the core temperature is higher in children than in young adults, because heat loss by greater cutaneous vasodilation cannot compensate for the underdeveloped sweat function.

\section{The decline of heat loss effector function with aging}

Sweat gland function decreases with aging. To determine how this occurs, we compared the $\mathrm{m}_{\mathrm{sw}}$ on the back and thigh between the older and young men during passive heating achieved by placing the lower legs and feet in a water bath at $42^{\circ} \mathrm{C}$ for $60 \mathrm{~min}$, while the subject was sitting in a controlled environment $\left(35^{\circ} \mathrm{C}, 45 \% \mathrm{RH}\right)$ (Inoue et al., 1991). The older men exhibited a sluggish $\mathrm{m}_{\mathrm{sw}}$ response on both sites, perhaps 


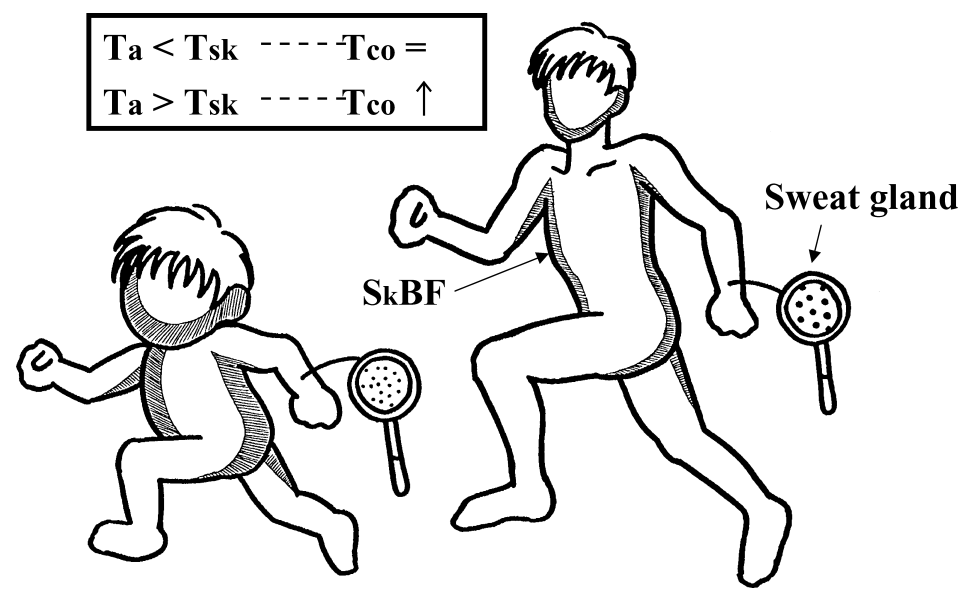

Fig. 1 The characteristics of heat loss responses in children. When the temperature of the air (Ta) is lower than that of the skin (Tsk), prepubertal children can regulate core temperature (Tco) as efficiently as young adults as a consequence of relatively greater cutaneous vasodilation on the head and trunk together with a greater surface area-to-mass ratio, despite having an underdeveloped sweat function. When the temperature of the air is higher than that of the skin, the greater surface area-to-mass ratio of children leads to a higher rate of heat absorption, because heat dissipation depends greatly on the evaporation of sweat. Under such conditions, the core temperature of children becomes higher than that of young adults, because heat loss by cutaneous vasodilation cannot compensate for the underdeveloped sweat function.

owing to decreased thermoreceptor sensitivity. During the latter half of the 60 -min passive heating period, $\mathrm{m}_{\mathrm{sw}}$ on the thigh was significantly lower among the older men than among the young men, but $\mathrm{m}_{\mathrm{sw}}$ did not differ on the back. Similar results were obtained using the same passive heating test during each of the four seasons (Inoue et al., 1995). These results suggest that aging-related decrements in sweat gland function may not occur at a uniform rate over the whole body.

In a longitudinal study between 1989 and 1994 (Inoue, 1996), the $\mathrm{m}_{\mathrm{sw}}$ on the back measured in 1994 was significantly lower than the value in 1989, whereas no such change occurred on the thigh. In a cross-sectional study using the same heating test and the same older men tested in 1994, the back and thigh $\mathrm{m}_{\mathrm{sw}}$ values were found to be significantly lower for the older men, while the $m_{\text {sw }}$ values on the forehead, chest, and forearm were not different between the older and young men (Inoue and Shibasaki, 1996). The decrement was markedly greater on the thigh than on the back. In a longitudinal study of four older men that spanned 10 years (Inoue, 2004), the $\mathrm{m}_{\mathrm{sw}}$ on the thigh (which was substantially lower than that of young men in 1989) was no different in 1989,1994 , and 1999 . The $\mathrm{m}_{\mathrm{sw}}$ on the back (which was similar to that of young men in 1989) decreased significantly from 1989 to 1994 , but did not change between 1994 and 1999. The $\mathrm{m}_{\mathrm{sw}}$ on the chest and forearm (which were similar to those of young men in 1994) decreased significantly from 1994 to 1999 , and the magnitude of the decrease was greater on the chest $\mathrm{m}_{\mathrm{sw}}$ than on the forearm $\mathrm{m}_{\mathrm{sw}}$. By contrast, the $\mathrm{m}_{\mathrm{sw}}$ on the forehead (which was similar to that of young men in 1994) increased significantly from 1994 to 1999. The results of these longitudinal and cross-sectional studies (Inoue et al., 1991, 1995; Inoue, 1996, 2004; Inoue and Shibasaki, 1996) suggest that the regional differences exist in the aging-related decrement in sweating and that this decrement may proceed sequentially from the lower limbs to the back of the upper body, then to the front of the upper body, thereafter to the upper limbs, and finally to the head.

The aging-related decrements in $\mathrm{m}_{\mathrm{sw}}$ on the thigh (Inoue et al., 1991, 1995) and on the thigh and back (Inoue and Shibasaki, 1996) in cross-sectional studies and in $\mathrm{m}_{\mathrm{sw}}$ on the back, chest, and forearm in longitudinal studies (Inoue, 1996, 2004) were the results of the decrease in SGO rather than a decrease in the recruitment of HASG. Based on the findings of Sato and Sato (1983) mentioned above, the decrease in SGO with aging might reflect aging-related changes in the sweat glands themselves (sweat gland atrophy) or a decrease in cholinergic sensitivity. Such changes may proceed gradually over the surface of the body to produce the distinctive regional differences that characterize the aging-related decrement in sweating.

In a longitudinal study, the density of HASG on the thigh (but not on the back) decreased significantly from 1989 to1994 (Inoue, 1996). Similarly, in a separate study, the density of HASG on the thigh and back decreased from 1994 to 1999 in association with a decrease in SGO (Inoue, 2004). By contrast, the density of HASG did not change on any body site in two cross-sectional studies (Inoue et al., 1991; Inoue and Shibasaki, 1996). It is possible that the progressive atrophy of sweat glands leads to a decrease in sweat output; that is, the aging-related decrement in SGO may occur before the HASG density is reduced. Similar SGO and HASG densities have been observed in older men stimulated by exercise and methylcholine (Inoue et al., 1999a; Inoue, 1999).

SkBF decreases with aging. In a cross-sectional study (Inoue and Shibasaki, 1996), we measured SkBF on the forehead, chest, and thigh, and calculated CVC by dividing SkBF by MAP for each body site. The increases in SkBF on 


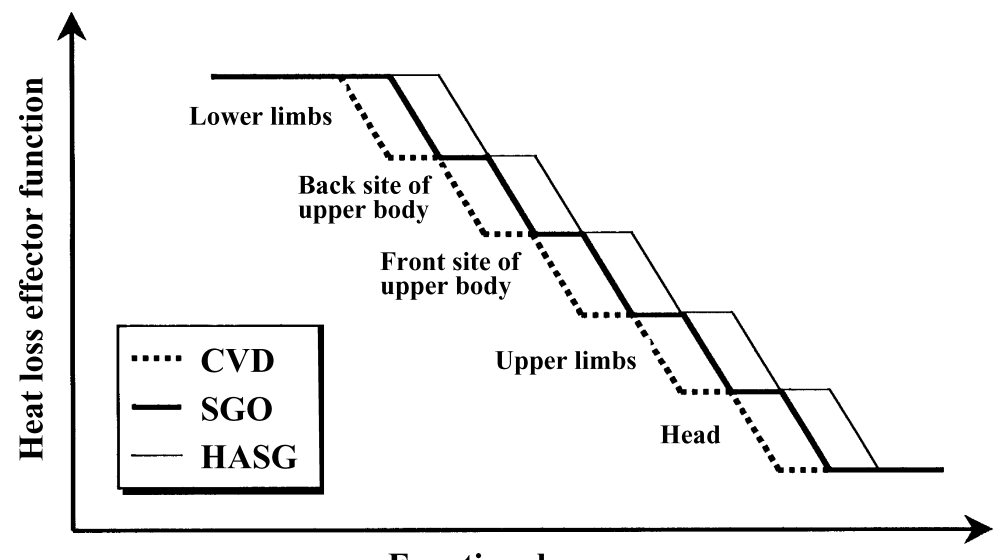

Functional age

Fig. 2 The effect of age on sweating and blood flow to the skin in response to heat. The age-related decrement in the heat loss effector function may first involve cutaneous vasodilation (CVD), then sweat output per gland (SGO), and finally active sweat gland density (HASG). The decrement may proceed initially from the lower limbs to the back of the upper body, to the front of the upper body, to the upper limbs, and finally to the head.

the chest and thigh were significantly less in older men during heat exposure, while the SkBF on the forehead was similar in both young and older men; although the older men had a somewhat delayed response (possibly owing to decreased thermoreceptor sensitivity). The lower SkBF values in the older men were more remarkable on the thigh rather than on the chest. The age-related differences in the CVC values at each site were similar to the SkBF responses, because the MAP responses were not affected by age or time. These observations indicate that the regional differences exist in the age-related decrement of SkBF in response to passive heating, which suggests that the decline may not occur at a uniform rate over the entire body surface. The percentages of total $\mathrm{m}_{\mathrm{sw}}$ and SkBF in the older men relative to young men during the last 30 min of heat exposure were 105 and $99 \%$ on the forehead, 99 and $58 \%$ on the chest, and 63 and $50 \%$ on the thigh, respectively. The decrement in SkBF was greater than that of $\mathrm{m}_{\mathrm{sw}}$, especially on the chest. These results suggest that an aging-related decrement in cutaneous vasodilation may precede the decrement in sweat gland function. Nevertheless, the aging-related declines in the cutaneous vasodilation and sweat functions may be interrelated: the decrease in the amount of oxygen supplied to the sweat glands, owing to a decrease in vasodilation, may lead to adaptive changes in the sweat glands themselves.

Figure 2 is a schematic representation of the effects of increasing age on sweating and blood flow to the skin, based on our cross-sectional and longitudinal findings. The agingrelated decrement in the heat loss effector function may first involve cutaneous vasodilation, then sweat output per gland, and finally active sweat gland density, and the decrement may proceed from the lower limbs to the back of the upper body, to the front of the upper body, then the upper limbs, and finally to the head.

\section{The primary mechanisms underlying maturation- and aging-related alterations in the heat loss function}

Cutaneous vasodilation: The SkBF in nonacral regions is under dual sympathetic nervous control by a noradrenergic vasoconstrictor system and an active vasodilator system. In nonacral areas, a small initial increase in SkBF is achieved by the release of vasoconstrictor tone, and a greater secondary increase in $\mathrm{SkBF}$ is accomplished by active vasodilation. As we have described previously (Inoue et al., 1998; Shibasaki et al., 1999), a plot of blood flow to the skin as a function of local sweat rate reveals three temporal phases in the relationship between $\mathrm{SkBF}$ and $\mathrm{m}_{\mathrm{sw}}$ : (1) an increase in SkBF with no increase in $\mathrm{m}_{\mathrm{sw}}$ (suggestive of a decrease in vasoconstriction), (2) an increase in $m_{s w}$ with no secondary increase in SkBF, and (3) an increase in both $\mathrm{SkBF}$ and $\mathrm{m}_{\mathrm{sw}}$ (suggestive of active vasodilation). The increase in SkBF in the first phase was significantly greater on the chest and back (but not on the forearm and thigh) of the boys compared with the young men, and it was lower on the forearm and thigh (but not on the chest and back) of the older men compared with the young men. In the third phase, the slope of the regression of SkBF on $\mathrm{m}_{\mathrm{sw}}$ was greater for the values on the chest and back (but not on the forearm and thigh) of the boys compared with the young men, and the slope was lower for values on the back, forearm, and thigh (but not on chest) of the older men compared with the young men. These results suggest that the greater SkBF observed on the forehead and trunk of boys and the lower SkBF of older men may be attributable to maturation- and aging-related alterations, respectively, of the vasoconstrictor relaxation and active vasodilation systems. In addition, it appears that such alterations do not occur at uniform rates over the surface of the body. Furthermore, the impairment of active vasodilation that occurs with aging may develop prior to any changes in the vasoconstrictor system.

We also compared the maximal cutaneous vascular 
conductance $\left(\mathrm{CVC}_{\max }\right)$ on the forehead, chest, back, forearm, and thigh among prepubertal boys, older men, and young men during $40 \mathrm{~min}$ of the local heating, to determine whether the physical changes in the structure of the cutaneous vasculature also contribute to the greater SkBF of the boys and the lower $\mathrm{SkBF}$ of the older men relative to the young men (Inoue, 1998). A local heating system kept the skin surface $\left(20 \mathrm{~cm}^{2}\right)$ at $42^{\circ} \mathrm{C}$ at all recording sites. The maximal $\mathrm{SkBF}$ was then divided by the MAP to yield $\mathrm{CVC}_{\max }$. We found that $\mathrm{CVC}_{\max }$ was significantly greater on the forehead, back and thigh (but not on the chest or forearm) of the boys compared with the young men, and it was lower on the forearm and thigh (but not on the forehead, chest, or back) of the older men compared with the young men. These results suggested that a modification of the cutaneous vasculature owing to maturationand aging-related differences in $\mathrm{CVC}_{\max }$ may contribute to the greater SkBF of the boys and the lower SkBF of the older men (relative to the young men) that we observed in previous studies.

Sweat function: Sweat is discharged in a pulsatile fashion from the sweat gland onto the skin surface. Direct evidence that sweat expulsion reflects centrally derived sudomotor activity was recently provided using microneurography to examine the relationship between the sympathetic activity in cutaneous nerves and pulsatile sweat output (Ogawa and Sugenoya, 1993). Via this approach, we examined the mechanism underlying the lower sweat rates of the children and older men (Shibasaki et al., 1997a; Inoue et al., 1999b). The slope of $m_{s w}$ vs. the frequency of sweat expulsion $\left(f_{s w}\right)$ was significantly lower on the chest, back, and thigh (but not on the forearm) of the boys compared with the young men and on the thigh (but not on the forehead, chest, back, or forearm) of the older men compared with the young men. No such difference existed for the slope of $\mathrm{f}_{\mathrm{sw}}$ vs. mean body temperature. These results suggested that the lower $\mathrm{m}_{\mathrm{sw}}$ of the boys and older men (relative to young men) is the result of the underdevelopment (in boys) or impairment (in older men) of peripheral sweating mechanisms, including the sweat glands and their surrounding tissues, rather than the underdeveloped or impaired central activity related to sudomotor function. These maturation- and aging-related alterations of peripheral sweating mechanisms were supported by the finding that the prepubertal boys and older men have a lower SGO compared with the young men during internal and external heat stress (see above) and during cholinergic stimulation (Inoue et al., 1999a). In a longitudinal study of the elderly over 10 years (Inoue, 2004), the forehead $\mathrm{m}_{\mathrm{sw}}$ increased significantly, while the $\mathrm{m}_{\mathrm{sw}}$ at other sites decreased, as mentioned above. This finding possibly implied that the central sudomotor activity in response to a given thermal input can be augmented to compensate for agingrelated impairment of peripheral mechanisms; this augmentation would take the form of an increase in $\mathrm{m}_{\mathrm{sw}}$ on the forehead without the impairment of peripheral mechanisms.

\section{The potential to modify maturation- and aging- related alterations in the heat loss function by exercise training}

Children: It is well known that exercise training contributes to increases in $\mathrm{M}_{\mathrm{sw}}, \mathrm{m}_{\mathrm{sw}}$, and $\mathrm{SkBF}$ at a given core temperature, and to a reduction in the salt concentration of sweat at a given $\mathrm{m}_{\mathrm{sw}}$, in young adults. Age-dependent differences in sweating in relation to exercise training were examined in a study by Araki et al. (1979). In that study, 168 children from 9 to 11 years old and 20 young adults (20 years old) ran outdoors for 5 min once a day, three or four times per week. (They were asked to run as fast as they could without stopping). This exercise regime was followed for 5-7 weeks. In addition, 83 children from 3 to 6 years old ran for $500 \mathrm{~m}$ in the same way as the former group, once a day, 2-5 times a week, for $8-16$ weeks. The distance that was covered in the $5 \mathrm{~min}$ of running tended to increase over time in each group. The $\mathrm{m}_{\mathrm{sw}}$ and the chloride concentration of sweat on the chest of the children were less affected by exercise than were the same parameters of young adults. We found that SkBF during passive heating was correlated with $\mathrm{VO}_{2} \max$ in prepubertal boys, whereas there was no correlation between $\mathrm{m}_{\mathrm{sw}}$ and $\mathrm{VO}_{2} \max$ (unpublished data). It is likely that exercise training improves the cutaneous vasodilation rather than increases the sweating in prepubertal children. Thus, the exercise training improves the heat loss responses in children; although it is unclear why the degree of the improvement seems to be less in children than in young adults.

The elderly: Regarding the research question "Can the aging-related decline in sweating and cutaneous vasodilation be slowed or reversed by the regular exercise", we recruited three groups: the young men (group $\mathrm{Y}$ : $\mathrm{VO}_{2} \mathrm{max}$, $47 \mathrm{ml} \cdot \mathrm{kg}^{-1} \cdot \mathrm{min}^{-1}$ ); older men of very fitness (group $\mathrm{HO}$ : $\left.48 \mathrm{ml} \cdot \mathrm{kg}^{-1} \cdot \mathrm{min}^{-1}\right)$; and the older men of average fitness (group NO: $30 \mathrm{ml} \cdot \mathrm{kg}^{-1} \cdot \mathrm{min}^{-1}$ ). The group HO was more active compared to the other groups, but all men were matched physically (Inoue et al., 1999a). The men were heat-acclimated by a daily $90-\mathrm{min}$ cycling exercise at $35 \% \mathrm{VO}_{2} \max$ in a hot environment $\left(43^{\circ} \mathrm{C}, 30 \% \mathrm{RH}\right)$ for 8 days. $\mathrm{M}_{\mathrm{sw}}$ during exercise in group NO was lower than in the other groups throughout the heat acclimation period $\left(\mathrm{M}_{\mathrm{sw}}\right.$ was similar in groups $\mathrm{HO}$ and $\left.\mathrm{Y}\right)$, although SGO in response to cholinergic stimulation was in the order $\mathrm{Y}>\mathrm{HO}>\mathrm{NO}$. These results suggested that regular exercise slows the aging-related decline in the heat loss effector function. This research finding is supported by the work of Inoue et al. (1994) who examined the relative influences of anthropometric measures, $\mathrm{VO}_{2} \mathrm{max}$, and pedometer readings in daily life on the heat responses of 20 older men using multiple regression analysis. Final $\mathrm{T}_{\mathrm{re}}, \mathrm{M}_{\mathrm{sw}}$, $\mathrm{SkBF}$ and CVC values were closely related to the pedometer readings and/or $\mathrm{VO}_{2}$ max.

Acknowledgments This work was supported by a Grantin-Aid for Scientific Research from the Ministry of Education, Science, Sports, and Culture of Japan (grant No. 16207022). 
We are grateful to our subjects for their cooperation.

\section{References}

Araki T, Toda Y, Matsushita K, Tsujino A (1979) Age differences in sweating during muscular exercise. J Physical Fitness Jpn 28: 239-248

Inoue Y (1996) Longitudinal effects of aging on heat-activated sweat density and output in healthy active older men. Eur J Appl Physiol 74: 72-77

Inoue Y (1998) Maximal cutaneous vascular conductance in prepubertal boys, young and older men and its regional differences. Descente Sports Science 19: 254-260 [In Japanese]

Inoue Y (1999) Regional differences in exercise-induced sweating and cutaneous vascular responses in young and older men. Descente Sports Science 20: 254-260 [In Japanese]

Inoue Y (2003) Heat loss effector function in children and the elderly. J Adaptation Med 7: 13-22 [In Japanese]

Inoue Y (2004) Changes in sweating response with age in healthy older men: 10-yr follow-up. (in preparation)

Inoue Y, Havenith G, Kenney WL, Loomis JL, Buskirk ER (1999a) Exercise- and methylcholine-induced sweating responses in older and younger men: effect of heat acclimation and aerobic fitness. Int J Biometeorol 42: 210216

Inoue Y, Nakao M, Araki T, Murakami H (1991) Regional differences in the sweating responses of older and younger men. J Appl Physiol 71: 2453-2459

Inoue Y, Nakao M, Okudaira S, Ueda H, Araki T (1995) Seasonal variation in sweating responses of older and younger men. Eur J Appl Physiol 70: 6-12

Inoue Y, Shibasaki M (1996) Regional differences in agerelated decrements of the cutaneous vascular and sweating responses to passive heating. Eur J Appl Physiol 74: 78-84

Inoue Y, Shibasaki M, Araki T (2002) Strategy for preventing heat illness in children and the elderly. In: Nose H, Gisolfi $\mathrm{CV}$, Imaizumi $\mathrm{K}$ eds. Exercise, Nutrition and Environmental Stress. Cooper Publishing Group, Traverse City (MI), 239271
Inoue Y, Shibasaki M, Hirata K, Araki T (1998) Relationship between skin blood flow and sweating rate, and age related regional differences. Eur J Appl Physiol 79: 17-23

Inoue Y, Shibasaki M, Ueda H, Ishizashi H (1999b) Mechanisms underlying the age-related decrement in the human sweating response. Eur J Appl Physiol 79: 121-126

Inoue Y, Ueda H, Nakao M, Araki T (1994) The relative influences of anthropometric measures, aerobic fitness, pedometer readings and microclimate temperatures in daily life on heat and cold responses of older men. Jpn J Biometeor 31: 189-199 [In Japanese]

Kondo N, Takano S, Aoki K, Shibasaki M, Tominaga H, Inoue $Y$ (1998) Regional differences in the effect of exercise intensity on thermoregulatory sweating and cutaneous vasodilation. Acta Physiol Scand 164: 71-78

Ogawa T, Sugenoya J (1993) Pulsatile sweating and sympathetic sudomotor activity. Jpn J Physiol 43: 275-289

Sato K, Sato F (1983) Individual variations in structure and function of human eccrine sweat gland. Am J Physiol 245: R203-208

Shibasaki M, Inoue Y, Kondo N (1997a) Mechanisms of underdeveloped sweating responses in prepubertal boys. Eur J Appl Physiol 76: 340-345

Shibasaki M, Inoue Y, Kondo N, Aoki K, Hirata K (1999) Relationship between skin blood flow and sweating rate in prepubertal boys and young men. Acta Physiol Scand 167: 105-110

Shibasaki M, Inoue Y, Kondo N, Iwata A (1997b) Thermoregulatory responses of prepubertal boys and young men during moderate exercise. Eur J Appl Physiol 75: 212218

Received: September 6, 2004

Accepted: September 30, 2004

Correspondence to: Yoshimitus Inoue, Laboratory for Human Performance Research, Osaka International University, 6-2157 Tohda-cho, Moriguchi, Osaka 570-8555, Japan

Phone: +81-6-6902-0791 (Ext. 2363)

Fax: +81-6-6902-8894

e-mail: inoueyos@hus.oiu.ac.jp 